\title{
Anatomía y Competencias Clínicas en Odontología. Estudio Basado en Apreciación de Académicos
}

\author{
Anatomy and Clinical Competences in Dentistry. Study Based on Academics Appreciation
}

*Víctor Aravena Verdejo \& **Fernando García-Hernández

ARAVENA, V. V. \& GARCÍA-HERNÁNDEZ, F. Anatomía y competencias clínicas en Odontología. Estudio basado en apreciación de académicos. Int. J. Morphol., 24(4):713-720, 2006.

RESUMEN: Los principales cambios que se están operando en la Educación Superior, corresponden a un replanteamiento de sus currículos, entre otros, la formación de profesionales con perfiles de egreso estructurados en base a competencias. El estudio de la Anatomía, no está ajena a estas innovaciones. Esta asignatura se dicta, tradicionalmente, en los primeros niveles de la formación profesional; sin embargo a la hora de aplicar losconocimientos en situaciones clínicas, éstos deben ser reincorporados o reforzados en los estudiantes, tanto por el tiempo transcurrido entre ambas situaciones de aprendizaje, como por la falta de relación y aplicación en el contexto clínico. En este sentido, cabe entonces preguntarse: ¿Cuáles son las competencias y conocimientos en el área de la Anatomía, que debe tener el estudiante, cuando se enfrente a situaciones clínicas, y qué grado de conocimientos reales posee?

El presente trabajo es un intento de determinar, por parte de 30 docentes de asignaturas clínicas del Departamento de Odontología de la Universidad de Antofagasta, Chile las competencias más relevantes que deben desarrollar los estudiantes en cada asignatura clínica, los conocimientos anatómicos requeridos para la ejecución de dichas competencias clínicas y evaluar el nivel de conocimientos anatómicos que manejan los estudiantes al momento de iniciar el aprendizaje de tales asignaturas. Se utilizó un cuestionario autoadministrado y se realizó una entrevista a cada Jefe de Unidad Clínica.

El 49,6\% de los docentes considera que los conocimientos anatómicos de los alumnos al iniciar las asignaturas clínicas, son muy buenos y buenos, el 42,5\% regulares y solamente el 7,9\% piensa que estos conocimientos son malos. Se determinaron las principales competencias que deben poseer los estudiantes en las distintas asignaturas clínicas y la relevancia de los conocimientos anatómicos necesarios para realizar procedimientos clínicos, así como los métodos sugeridos para reforzar la anatomía.

\section{PALABRAS CLAVE: Anatomía; Odontología; Educación.}

\section{INTRODUCCIÓN}

La Anatomía es una de las disciplinas básicas que contribuye a la formación de los estudiantes de Ciencias de la Salud, entregando conceptos relevantes así como una nomenclatura o terminología fundamental de aplicación a otras asignaturas básicas y especialmente de clínicas. Por esta razón, desde tiempos pretéritos esta disciplina se ubica temporalmente en los primeros años de docencia delas carreras del Área de la Salud, no escapando a lo anterior la Odontología aunque, desde mediados del siglo pasado, se recomienda la verticalización de conceptos anatómicos de aplicación en las asignaturas profesionales (Comité de Expertos de la OMS/OPS en la Enseñanza de la Morfología, 1970; DiDio, 1973; Sociedad Anatómica Española, 1996 y Universitat de Barcelona. Facultad de Medicina.
Nuevas tendencias en Educación Superior expresadas en documentos gubernamentales y de organismos internacionales, han conducido a un replanteamiento de los currículos universitarios (CINDA, 2004).

No es de extrañar entonces que en universidades norteamericanas como Harvard y MacMasters y europeas como Maastrich se desarrollara la metodología de aprendizaje basado en problemas (ABP) que modifica la enseñanza de la Anatomía integrándola no sólo con disciplinas afines sino que también con disciplinas preclínicas y clínicas a lo largo de todos años de estudio de la Carrera, metodología pedagógica que posteriormente se empezó a aplicar en distintas instituciones de todos los continentes con singular éxito. Va-

\footnotetext{
* Departamento de Odontología, Facultad de Medicina y Odontología, Universidad de Antofagasta, Chile.

** Unidad de Anatomía Departamento Biomédico, Facultad de Ciencias de la Salud, Universidad de Antofagasta, Chile.
} 
riantes de esta metodología son el aprendizaje basado en la evidencia, últimamente el aprendizaje basado en las tareas (TBL), y la educación médica basada en las competencias, ambas desarrolladas por Harden y colaboradores en la Universidad de Dundee en Escocia, Reino Unido (European Dental Education, 2000; Universitat de Barcelona, op. cit; Federación Dental Internacional, 2003; Universidad de Antofagasta; Modelo Pedagógico Universidad de Antofagasta (2005).

Educadores europeos han definido competencia como: "una capacidad individual para realizar un conjunto de tareas o de operaciones y el principio de la regulación por normas o estándares de calidad". Irigoin, por su parte, define competencia como "un conjunto identificable de conocimientos, actitudes, valores y habilidades relacionadas entre sí que permiten desempeñar satisfactoriamente en situaciones reales de trabajo, según estándares utilizados en el área ocupacional" (CINDA, 2004).

La educación basada en las competencias enfatiza el producto final y define lo que obligatoriamente debe saber el alumno al finalizar su formación, independiente de cómo lo enseñó el profesor o de los sistemas de aprendizaje que utilizó. Las competencias finales determinan lo que se ha de enseñar y permiten identificar lo esencial de lo superfluo. Sin lugar a dudas, representa el mejor método para acreditar la calidad del "producto elaborado" por cada institución de Educación Superior, representado por el profesional que se envía a la sociedad para que cumpla el rol que corresponde a su profesión en la comunidad. (Brailovsky, 2002).

En Septiembre de 2003, la Asamblea General de la Federación Dental Internacional (FDI) aprobó el documento "Resultados recomendados (competencias) en el entrenamiento de estudiantes de pregrado", en cuya introducción señala textualmente: "el término competencia clínica se refiere a una combinación de habilidades, actitudes y conocimientos que provee al clínico de suficiente competencia para emprender una tarea clínica específica. El conocimiento requerido comprende el entendimiento apropiado de los principios biológicos moleculares, a través de características anatómicas y fisiológicas hasta la patogenia de la enfermedad. Esto no es simplemente una capacidad técnica o cierta cantidad de conocimiento prescrito, esto implica mucho más. La adquisición de competencia clínica puede lograrse por medio de la diversidad existente en los programas de entrenamiento y enseñanza, los cuales pueden ser valorados y examinados de diferente manera en los distintos centros de Enseñanza Superior. No obstante, es posible llegar a un acuerdo sobre ciertas competencias clínicas básicas comunes en todos los miembros de la profesión dental".
En este contexto, la FDI recomienda que para cualquier competencia quirúrgica dental debe poseerse sólidos conocimientos de los principios biológicos, fisiológicos y anatómicos requeridos para completar certeramente el procedimiento.

Cada institución de Educación Superior debe definir el perfil profesional que desea que sus egresados posean al salir al medio laboral y, que de alguna manera, representa la impronta formativa lograda al finalizar sus estudios de pregrado.

La Comisión Nacional de Acreditación del Pregrado (CNAP) y la Asociación Chilena de Escuelas de Odontología (ACHEO) han elaborado competencias generales y específicas que deben reflejarse en el perfil profesional del egresado de Odontología.

En este sentido, la Carrera de Odontología de la Universidad de Antofagasta, en el Plan de Estudios de la Carrera, formuló su perfil profesional. Sin embargo, cabe preguntarse ¿cuáles son las competencias y conocimientos en el área de la Anatomía, que debe tener el estudiante, cuando se enfrente a situaciones clínicas, por un lado y qué grado de conocimientos reales posee, cuando se enfrenta a disciplinas clínicas?

El propósito de la presente investigación es obtener información de parte de los docentes que imparten dichas asignaturas, referente a su apreciación respecto a: 1) las principales competencias más relevantes de cada asignatura clínica, 2) los conocimientos anatómicos requeridos para la ejecución de dichas competencias clínicas durante el entrenamiento del pregrado y 3) valorar el nivel de conocimientos anatómicos que manejan los estudiantes al momento de iniciar la docencia en sus respectivas asignaturas.

\section{MATERIAL Y MÉTODO}

La población de estudio corresponde a 30 académicos de asignaturas clínicas de la Carrera de Odontología, de la Universidad de Antofagasta, lo cual representa el 50\% del total de docentes que participan en la enseñanza de asignaturas clínicas, desde cuarto a sexto año de la carrera, con el propósito de establecer, según su criterio: a) las competencias más relevantes de cada asignatura clínica, b) los conocimientos anatómicos requeridos para la ejecución de dichas competencias clínicas durante el entrenamiento del pregrado y c) valorar el nivel de conocimientos anatómicos que manejan los estudiantes al momento de iniciar la docencia en sus respectivas asignaturas. 
Para este efecto, se elaboraron dos instrumento de recolección de datos: 1) uno de tipo cuestionario autoadministrado, diferenciado para cada nivel, confiable $\mathrm{y}$ validado, mediante preguntas abiertas y preguntas cerradas con escala de preferencia, que se aplicó a todos los docentes clínicos de la Carrera, y 2) un instrumento elaborado con directrices que sirva de pauta para realizar entrevistas a cada uno de los jefes de las unidades clínicas, entrevistas que fueron grabadas. Los antecedentes así recogidos, fueron vertidos en una ficha que permitió su ordenamiento.

Una vez tabulados y ordenados los datos recopilados en los cuestionarios, se efectuó análisis estadístico mediante programas Microsoft Office Excel 7.0 y Statgraphics 4.0.

\section{RESULTADOS Y DISCUSIÓN}

El análisis de los resultados fue dividido en dos grupos. El primero, detalla los datos obtenidos en la encuesta aplicada a los académicos de la Carrera de Odontología, que estuvieron dispuestos a entregar información referida a competencias clínicas y anatómicas que deben poseer los estudiantes al finalizar la asignatura de su responsabilidad, así como también, los conocimientos anatómicos requeridos, su apreciación del conocimiento anatómico que poseían los alumnos al iniciar cada asignatura clínica y los métodos usados para reforzar los conocimientos insatisfactorios para el desempeño clínico. El segundo grupo de resultados analiza los aspectos conceptuales obtenidos en las entrevistas realizadas a académicos jefes de unidad o de clínica.

En la encuesta autoadministrada a los 30 académicos de asignaturas clínicas, y de acuerdo a los propósitos de este trabajo, se buscaba obtener cuatro datos distintos: 1) las competencias clínicas que deben poseer los estudiantes al término de cada asignatura, 2) los conocimientos anatómicos necesarios para dichas competencias, 3) la opinión del nivel de conocimiento anatómico que poseen los alumnos al iniciar las asignaturas clínicas, y 4) métodos de reforzamiento y repaso de los conocimientos anatómicos aprendidos en el primer nivel, en caso de considerar insuficientes o débiles dichos conocimientos.

Tabla I. Competencias clínicas y conocimientos anatómicos relevantes, expresados en porcentaje, requeridos para la realización de procedimientos clínicos. Encuesta autoadministrada a académicos del Departamento de Odontología de la Universidad de Antofagasta, Chile ( $\mathrm{n}=30)$. (valores ).

\begin{tabular}{|c|c|c|c|c|c|c|c|c|c|c|c|c|c|}
\hline & $\begin{array}{l}\text { Competencias } \\
\text { clínicas } \\
\text { (expresado en } \\
\text { porcentaje) }\end{array}$ & $\begin{array}{l}\text { Esqueleto } \\
\text { Maxilo } \\
\text { facial }\end{array}$ & $\begin{array}{l}\text { Neuro- } \\
\text { muscular }\end{array}$ & Vascular & Visceral & Glandular & ATM & $\begin{array}{l}\text { Boca y } \\
\text { dientes }\end{array}$ & $\begin{array}{l}\text { Topog. } \\
\text { cabeza y } \\
\text { cuello }\end{array}$ & $\begin{array}{l}\text { Plano } \\
\text { cutáneo }\end{array}$ & $\begin{array}{c}\text { Reparos } \\
\text { anatómicos }\end{array}$ & $\begin{array}{c}\text { Anatomía } \\
\text { Radiológica }\end{array}$ & Nomenclatura \\
\hline 1 & $\begin{array}{l}\text { Anamnesis, } \\
\text { examen clínico y } \\
\text { ficha clínica } \\
(43,3 \%)\end{array}$ & $\underline{\mathbf{5 0 , 0}}$ & 40,0 & 33,3 & 30,0 & 33,3 & 43,3 & 43,3 & 36,7 & 30,0 & 43,3 & 46,7 & 40,0 \\
\hline 2 & $\begin{array}{l}\text { Diagnóstico } \\
\text { diferencial entre } \\
\text { normal y } \\
\text { patológico } \\
(43,3 \%)\end{array}$ & $\underline{66.7}$ & 46,7 & 43,3 & 56,7 & 60,0 & 46,7 & 60,0 & 43,3 & 46,7 & 53,3 & 60,0 & $\underline{66,7}$ \\
\hline 3 & $\begin{array}{l}\text { Rehabilitación oral } \\
(40,0 \%)\end{array}$ & 26,7 & 30,0 & 3,3 & 3,3 & 6,7 & 30,0 & $\underline{43,3}$ & 10,0 & 6,7 & 23,3 & 23,3 & 23,3 \\
\hline 4 & $\begin{array}{l}\text { Interpretación de } \\
\text { imágenes } \\
\text { radiográficas } \\
(33,3 \%)\end{array}$ & 43,3 & 30,0 & 30,0 & 30,0 & 26,7 & 33,3 & 46,7 & 30,0 & 30,0 & 40,0 & $\underline{76,7}$ & 40,0 \\
\hline 5 & $\begin{array}{l}\text { Plan de } \\
\text { tratamiento según } \\
\text { normas clínicas y } \\
\text { éticas }(30,0 \%)\end{array}$ & $\underline{\mathbf{3 0}, 0}$ & 20,0 & 20,0 & 3,3 & 10,0 & 16,7 & 26,7 & 10,0 & 13,3 & 26,7 & 10,0 & 6,7 \\
\hline 6 & $\begin{array}{l}\text { Capacidad de } \\
\text { formular un } \\
\text { pronóstico } \\
(30,0 \%)\end{array}$ & 13,3 & 10,0 & 10,0 & 16,7 & 16,7 & 10,0 & $\underline{20,0}$ & 10,0 & 16,7 & 10,0 & 10,0 & $\underline{20,0}$ \\
\hline 7 & $\begin{array}{l}\text { Manejo de } \\
\text { urgencias } \\
(26,7 \%)\end{array}$ & 16,7 & 20,0 & 20,0 & 10,0 & 10,0 & 6,7 & 20,0 & 20,0 & 10,0 & 20,0 & 20,0 & $\underline{23,3}$ \\
\hline 8 & $\begin{array}{l}\text { Manejo de } \\
\text { técnicas } \\
\text { anestésicas } \\
(23,3 \%)\end{array}$ & 26,7 & 36,7 & 26,7 & 13,3 & 26,7 & 16,7 & 30,0 & 26,7 & 16,7 & $\underline{40,0}$ & 20,0 & 23,3 \\
\hline 9 & $\begin{array}{l}\text { Restauración } \\
\text { estructuras } \\
\text { dentarias perdidas } \\
(23,3 \%)\end{array}$ & 23,3 & 13,3 & 6,7 & 6,7 & 6,7 & 16,7 & $\underline{40,0}$ & 13,3 & 6,7 & 23,3 & 23,3 & 23,3 \\
\hline 10 & $\begin{array}{l}\text { Manejo clínico y } \\
\text { farmacológico del } \\
\text { dolor }(20 \%)\end{array}$ & 20,0 & $\underline{36,7}$ & 26,7 & 13,3 & 26,7 & 16,7 & $\underline{\mathbf{3 0 , 0}}$ & 26,7 & 16,7 & 33,3 & 20,0 & 20,0 \\
\hline
\end{tabular}


La Tabla I detalla las principales competencias clínicas que deben adquirir los estudiantes de Odontología al finalizar sus estudios de pregrado y los conocimientos anatómicos fundamentales que deben poseer los estudiantes para realizar los procedimientos clínicos necesaria para casa competencia.

Considerando que las competencias clínicas dependen de la disciplina que se trate, se observó una multiplicidad de respuestas, algunas de ellas muy específicas y relacionadas, más que con el aprendizaje de pregrado, con conocimientos propios del postgrado de la especialidad.

La Fig. 1 muestra el porcentaje de veces que un conocimiento anatómico fue citado por los académicos en las encuestas, independiente, que el tipo de compendia clínica fuese relevante o general.

En la Fig. 2 se compara el conocimiento anatómico básico para realizar las competencias relevantes señaladas por los académicos, con el conocimiento anatómico requerido para realizar todas las competencias clínicas de la carrera, demostrándose una marcada correlación.

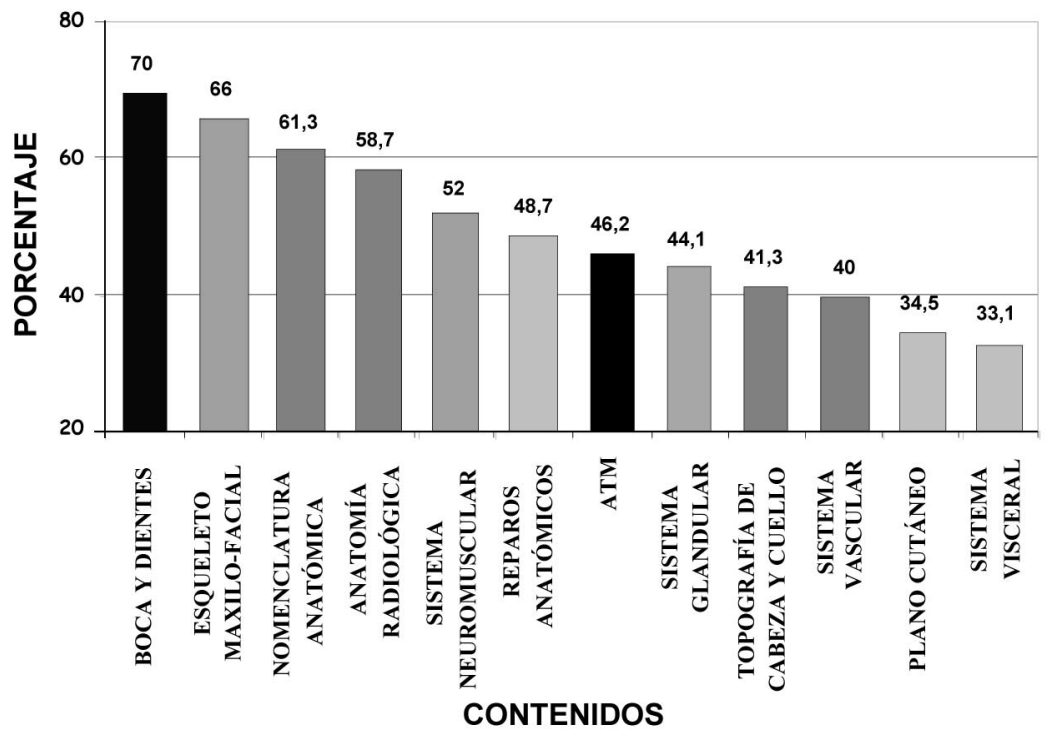

Fig. 1. Porcentaje de conocimientos anatómicos necesarios para lograr competencias clínicas según académicos del Departamento de Odontología de la Universidad de Antofagasta, Chile $(\mathrm{n}=30)^{*}$.

En la Fig. 3 se grafica la apreciación porcentual de los académicos clínicos respecto al nivel de conocimiento anatómico que poseen los estudiantes al iniciar las asignaturas clínicas.

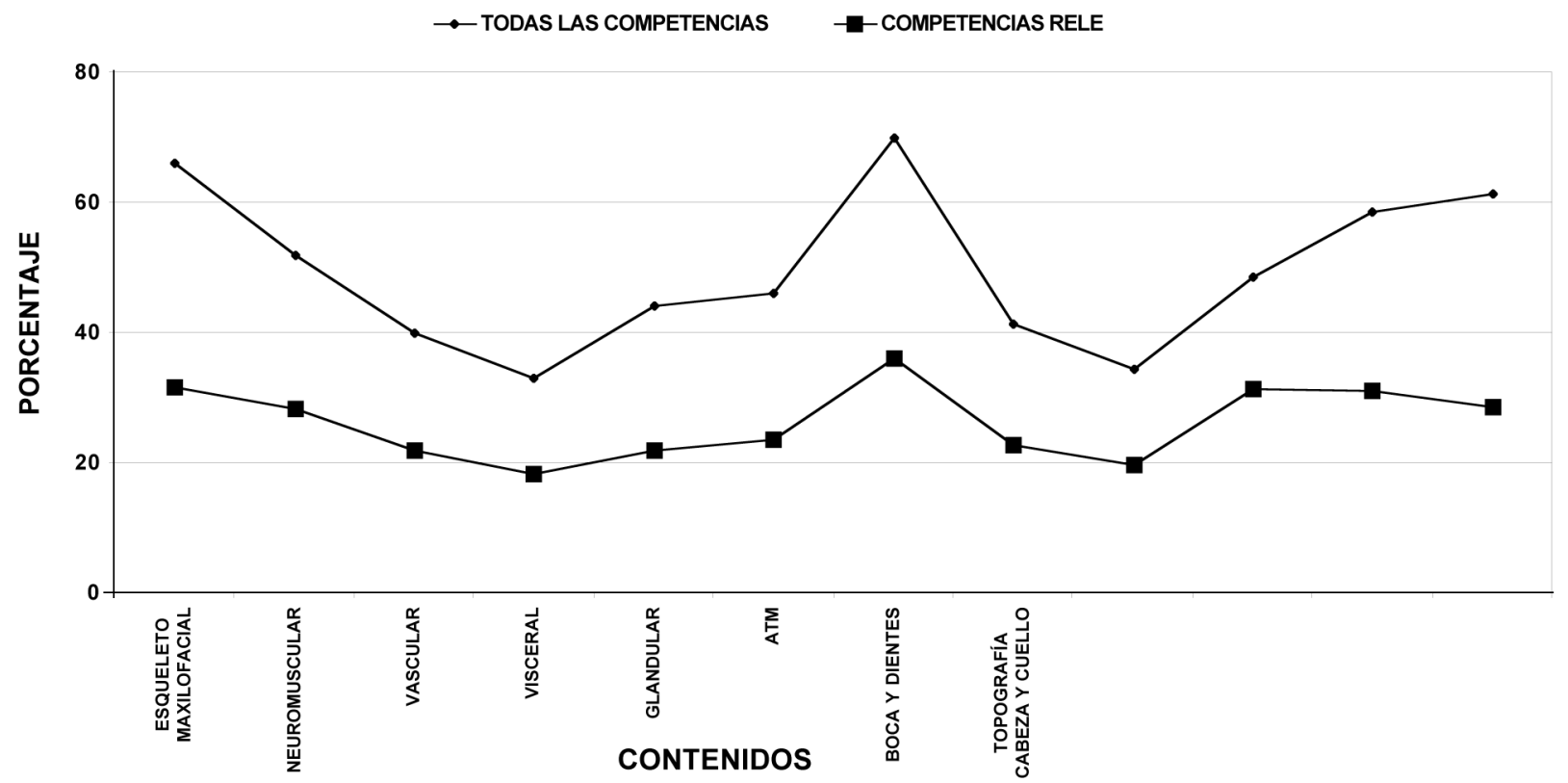

Fig. 2. Comparación de porcentajes de conocimientos anatómicos citados, necesarios para realizar competencias clínicas generales y competencias relevantes. Encuesta autoadministrada a académicos del Departamento de Odontología, Universidad de Antofagasta, Chile $(\mathrm{n}=30)$. Fuentes: Tabla I y Fig. 1. 
La Fig. 4 muestra la correlación existente entre la apreciación de los académicos sobre el porcentaje de conocimiento anatómico específico que manejan los estudiantes (ver Fig. 1), con los conocimientos necesarios para realizar los procedimientos clínicos, determinándose una correlación del $82 \%$ entre los conocimientos esperados y los conocimientos que poseen los estudiantes.
La Fig. 5 grafica la posición o ranking que los académicos del Departamento de Odontología asignan a cada grupo de conocimientos anatómicos según su relevancia para realizar los procedimientos clínicas de acuerdo a las competencias que los estudiantes deben poseer al finalizar las diversas asignaturas clínicas versus la apreciación del nivel de conocimientos que poseen los estudiantes al inicio de las asignaturas clínicas.

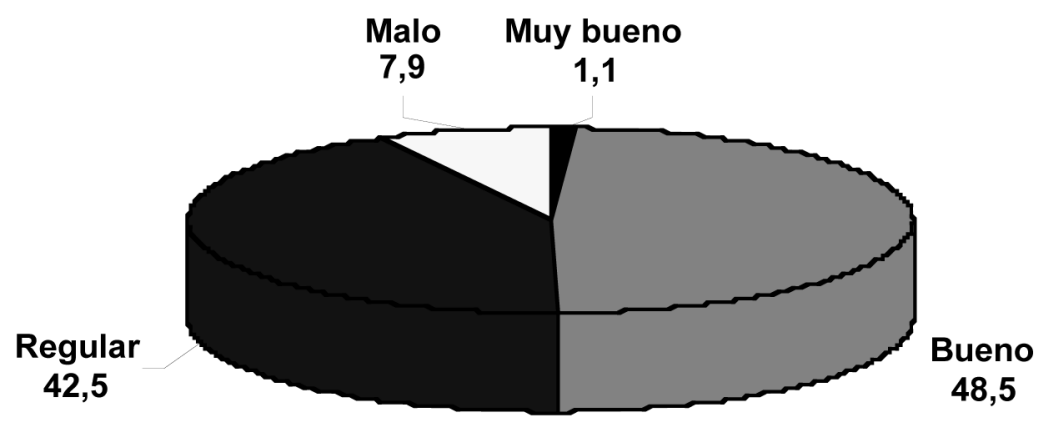

Fig. 3. Porcentaje del nivel de conocimiento anatómico que poseen los estudiantes al iniciar las asignaturas clínicas, según apreciación de los académicos del Departamento de Odontología, Universidad de Antofagasta $(\mathrm{n}=30)^{*}$.

* El valor porcentual se obtuvo por el número de citas de los conocimientos requeridos para las competencias (número máximo de citas por académico, de cada uno de los conocimientos $=5$ ).

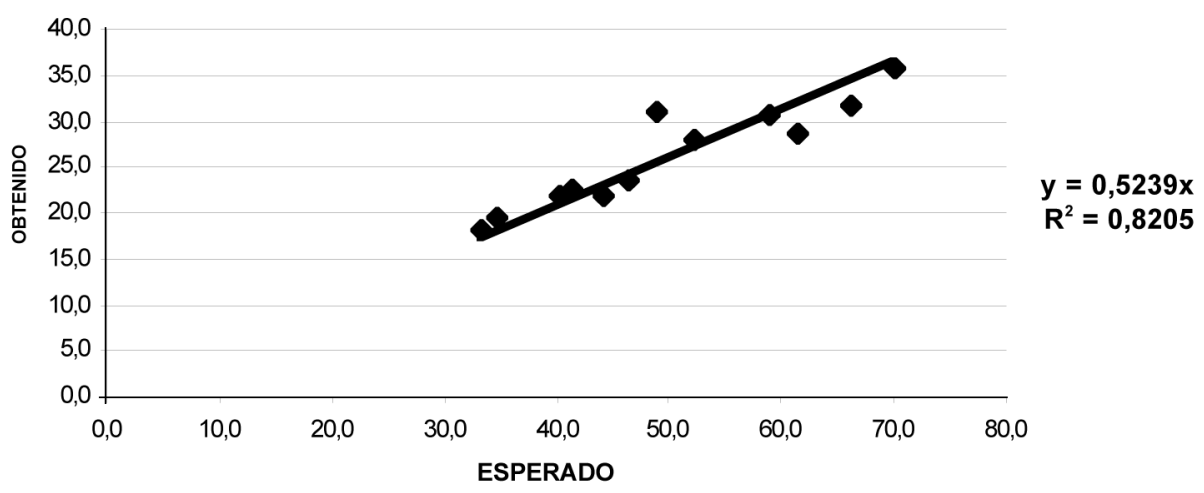

Fig. 4. Regresión lineal que muestra la correlación entre los conocimientos requeridos y los que presentan los alumnos de Odontología, al momento de iniciar la clínica, según apreciación de los académicos del Departamento de Odontología de la Universidad de Antofagasta. $(n=30)$.

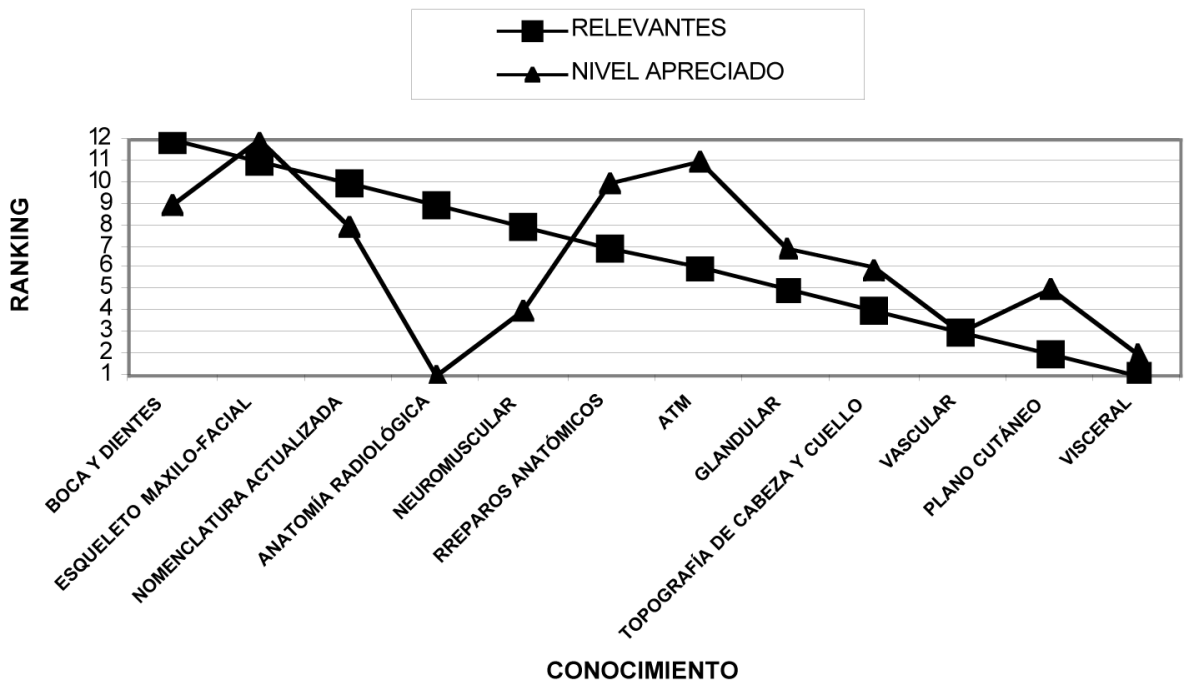

Fig. 5. Comparación entre conocimiento anatómico que posee el alumno al inicio de actividades clínicas y los conocimientos requeridos para realizar todas las competencias clínicas, según la opinión de los académicos del Departamento de Odontología de la Universidad de Antofagasta. $(n=30)$. 
En la Fig. 6 se detalla el porcentaje de los métodos predilectos utilizados por los académicos de asignaturas clínicas para reforzar y actualizar los conocimientos anatómicos olvidados por los estudiantes de la carrera de Odontología y que se requieren para realizar los diversos procedimientos clínicos establecidos para cada competencia clínica.

Del análisis de la entrevista aplicada a profesores jefes de unidades clínicas y/o jefes de clínicas de asignaturas profesionales de la carrera de Odontología, se comprobó que la mayoría de los profesores opinan lo siguiente: 1) los alumnos que inician las asignaturas clínicas poseen una adecuada preparación anatómica para desarrollar los procedimien- tos clínicos; 2) la nomenclatura anatómica actual es desconocida por la mayor parte del profesorado; 3) se utilizan diversos métodos de reforzamiento de los conocimientos anatómicos necesarios para realizar cada procedimiento clínico, mediante demostraciones prácticas, análisis de casos clínicos y, fundamentalmente, mediante el repaso constante de literatura anatomo-clínica; 4) todos los profesores coinciden en que los contenidos de Anatomía de Cabeza y Cuello y Anatomía Bucodentaria, que se dictan como parte de la asignatura de Anatomía Humana en el primer nivel de la Carrera, deberían formar parte de una asignatura de Anatomía Clínica Odontológica, que tendría que ser dictada a nivel de tercer año del plan de estudios, es decir, en un nivel próximo a la real aplicación de esos conocimientos.

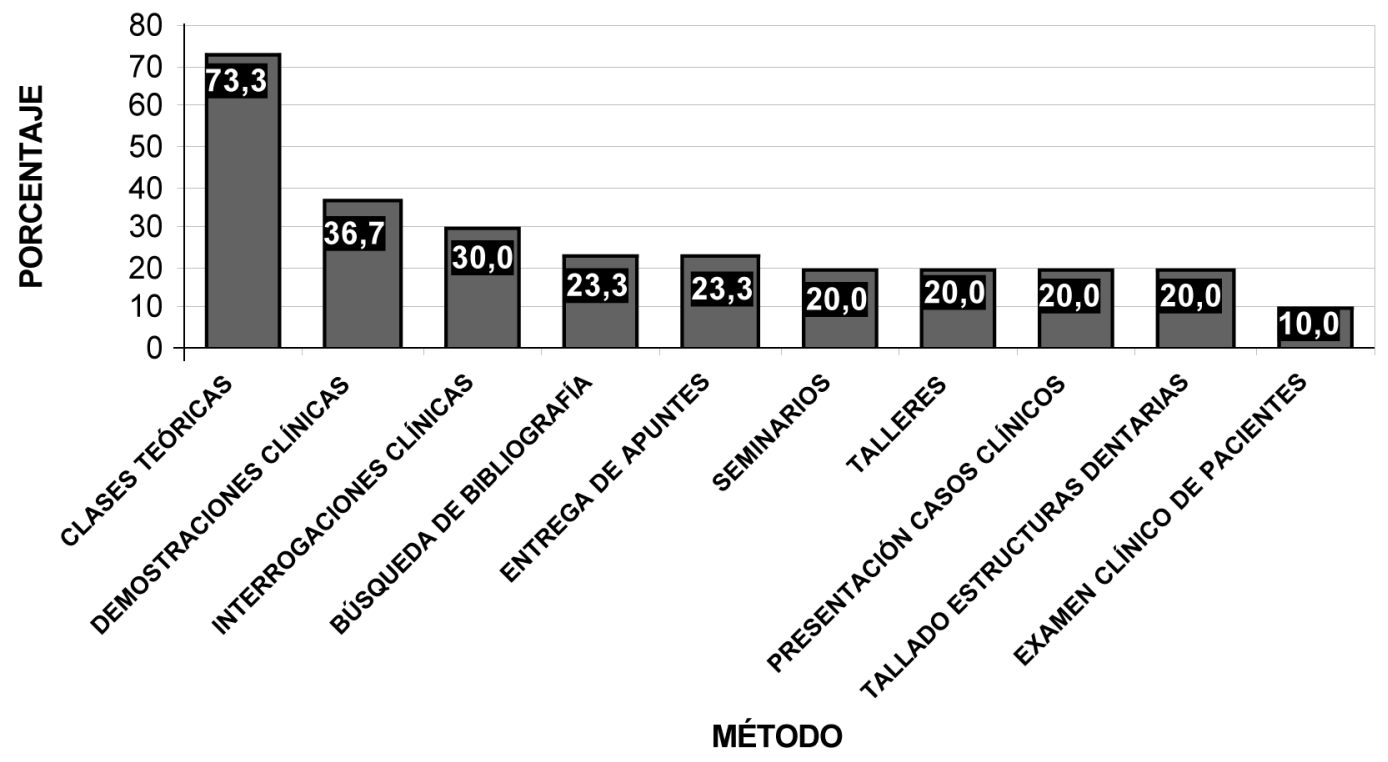

Fig. 6. Ranking comparativo de conocimientos anatómicos considerados como relevantes para las competencias clínicas versus apreciación del nivel de los conocimientos de los estudiantes, según la opinión de los académicos del Departamento de Odontología de la Universidad de Antofagasta. $(n=30) *$.

* Para establecer el ranking se consideró, por un lado, solamente el porcentaje de conocimientos anatómicos que poseen los estudiantes al iniciar asignaturas clínicas, apreciado por los académicos como muy bueno y bueno y, por otra parte, el porcentaje en que dicho conocimiento fue citado como necesario para realizar cualquier tipo de procedimiento clínico en las diversas competencias y que debe poseer el estudiante de Odontología al finalizar sus estudios de pregrado.

\section{CONCLUSIONES}

Del análisis de los resultados obtenidos y detallados en los gráficos anteriormente expuestos, se pueden extraer las siguientes conclusiones:
1) El $49,6 \%$ de los académicos encuestados considera que los conocimientos anatómicos que poseen los alumnos, al iniciar las asignaturas clínicas, son muy buenos y buenos. 
Ratificando lo anterior, la mayoría de los profesores entrevistados considera que el nivel y profundidad de los conocimientos anatómicos son adecuados para desarrollar las diversas competencias clínicas.

2) Se determinó el orden prioritario de relevancia de los conocimientos anatómicos necesarios para realizar procedimientos clínicos, comprobándose también un nivel general aceptable de conocimientos que poseen los estudiantes al inicio de los cursos clínicos, siendo la mayor parte de éstos superior a los requerimientos; sin embargo, los conocimientos de boca y dientes, neuromuscular, nomenclatura y, muy en especial, anatomía radiológica están muy por debajo de las necesidades mínimas requeridas en clínica. Estos antecedentes recomiendan modificar a futuro los contenidos y profundidad de algunos capítulos de la disciplina anatómica a fin de cautelar las necesidades de las asignaturas clínicas, lo cual coincide con la opinión de uno de los profesores entrevistados.
3) Los profesores entrevistados recomiendan la creación de una asignatura de Anatomía Clínica Odontológica en el tercer nivel de la Carrera, que entregue los conocimientos de anatomía de cabeza y cuello requeridos por las diversas asignaturas clínicas.

4) Los académicos priorizan la realización de clases teóricas y de demostraciones clínicas como medio de reforzamiento. En relación a lo anterior, los profesores entrevistados solucionan el eventual olvido mediante la realización de clases teóricas, demostraciones y análisis de casos clínicos, recomendando, además un permanente repaso bibliográfico por parte de los estudiantes antes de realizar los procedimientos clínicos.

5) Se logró obtener un listado de las 10 competencias clínicas más relevantes, las cuales deben poseer los estudiantes de Odontología al finalizar los estudios de las diversas asignaturas clínicas durante su formación de pregrado.

ARAVENA, V. V. \& GARCÍA-HERNÁNDEZ, F. Anatomy and clinical competences in Dentistry. Study based on academics appreciation. Int. J. Morphol., 24(4):713-720, 2006.

SUMMARY:The main changes that are taking place in the superior education correspond to a reframing of their curricula. Among others, the formation of professionals with end profiles structured on the basis of competences. The study of the anatomy is not far to these innovations. This is dictated, traditionally, in the first levels of the professional formation; nevertheless at the time of applying them in clinical situations, this knowledge must be reincorporated or reinforced in the students, on the one hand, by the time passed between both situations of learning and by another one by the lack of relation and application in the clinical context. In this sense, it is possible then to ask itself: Which are the competences and knowledge in the area of the anatomy, that must have the student when it faces clinical situations, and what degree of real knowledge has?

The present work is an attempt to determine on the part of 30 teachers of clinical subjets of the Department of Dentistry of the University of Antofagasta the more relevant competences than must develop the students in each clinical subjet, the anatomical knowledge required for the execution of these clinical competences and to evaluate the level of anatomical knowledge that handle the students at the time of initiating the learning of such subjets. A self-administered questionnaire was used and an interview to each clinical unit chief was made.

The $49.6 \%$ of the teachers consider that the anatomical knowledge of the students when initiating the clinical subjets are very good and good, regular $42.5 \%$ and only $7.9 \%$ think that these knowledge are bad. The main competences that must have the students in the different clinical subjets and the relevance from the anatomical knowledge necessary to make clinical procedures, as well as the suggested methods were determined to reinforce the Anatomy.

KEY WORDS: Gross Anatomy; Dentistry; Education.

\section{REFERENCIAS BIBLIOGRÁFICAS}

Brailovsky, C. A. Educación Médica, evaluación de las competencias. 2002. http://www.fmv.uba.org.ar/proaps/9pdf

CINDA- Centro Interuniversitario de Desarrollo. Competencia de Egresados Universitarios, Santiago, 2004.

Comité de Expertos de la OMS/OPS en la Enseñanza de la
Morfología. Enseñanza de la Morfología en las Escuelas de Medicina de la América Latina. Primer Informe. Serie Desarrollo de Recursos Humanos A. Educación Médica No 9, Organización Panamericana de la Salud, Oficina Sanitaria Panamericana, Oficina Regional de la ORGANIZACIÓN MUNDIAL DE LA SALUD, Washington D. C., 1970. 
DiDio, L. J. A. Tendencias modernas en la educación médica y su repercusión en la enseñanza de la Anatomía. Educación médica y salud, 2:184-97, 1973.

European Dental Education. Core Knowledge, 2000. www.dented.org/parse.php3 ?file =content/ knowledge.html

Federación Dental Internacional. Declaración de la FDI: Resultados (competencias) en el entrenamiento de estudiantes de pregrado. www:|Exec OfficelStatements| Approved by GAISpanish\Basic dental Training - SP Clinical Competences 2003 Version dated,18 September 2003.

Sociedad Anatómica Española. II Jornadas sobre Enseñanza de la Anatomía. Bases para los programas en los planes de estudio de las diferentes Licenciaturas y Diplomaturas. Patrocinado por Sociedad Anatómica Española. Universidad Complutense, Facultad de Medicina, Madrid 10 de Mayo de 1996.

Universidad de Antofagasta, Vicerrectoría Académica. Modelo Pedagógico de la Universidad de Antofagasta, 2005.

Universitat de Barcelona. Facultad de Medicina. Competencias a adquirir por los estudiantes de Medicina durante el pregrado en la Facultad de Medicina de la Universitat de Barcelona. www.ub.es/medicina/castella/ compcast.pdf
Dirección para correspondencia: Prof. Dr. Fernando García Hernández Unidad de Anatomía. Departamento Biomédico Facultad de Ciencias de la Salud Universidad de Antofagasta CHILE

Email:fgarcia@uantof.cl

Recibido : 29-05-2006

Aprobado: 27-09-2006 